TRANSACTIONS OF THE

AMERICAN MATHEMATICAL SOCIETY

Volume 349, Number 7, July 1997, Pages 2605-2617

S 0002-9947(97)01567-5

\title{
ANALYTIC SUBGROUPS OF $t$-MODULES
}

\author{
ROBERT TUBBS
}

\begin{abstract}
In this paper we study the structure of analytic subgroups and of $t$-submodules of $t$-modules.
\end{abstract}

\section{INTRODUCTION}

In $[\mathrm{An}] \mathrm{G}$. Anderson introduced the notion of an abelian $t$-module in order "to answer two questions about V.G. Drinfeld's elliptic $A$-modules and certain higherdimensional generalizations." One of these dealt with reconciling two approaches to the construction of elliptic $A$-modules: one of them being through lattices and the other through "shtukas." The other question concerned generalizations of elliptic $A$-modules which are analogues of Hilbert-Blumenthal abelian varieties. The categories that Anderson discovered in answering these questions are the anti-equivalent categories of abelian $t$-modules and $t$-motives.

An abelian $t$-module (defined below) provides a (possibly) higher dimensional analogue of a Drinfeld elliptic module. Associated with Drinfeld elliptic modules is a rich transcendence theory which is primarily due to J. Yu. In a series of papers dating back to $1985 \mathrm{Yu}$ developed both a classical and modern transcendence theory for these objects. Before Yu, however, L.I. Wade had established the transcendency of certain numbers associated with a Carlitz module (which is a special type of Drinfeld elliptic module).

The goal of this paper is to establish results for abelian $t$-modules which are fundamental to the study of their modern transcendency aspects. Yu has already laid some of these foundations, and it seems appropriate to further examine the relevant properties of the category of abelian $t$-modules without an immediate regard for the arithmetic applications. In a sequel to this paper we will establish the transcendence consequences of these results.

A unifying element of modern transcendence theory has been that of an analytic homomorphism from a complex vector space into an algebraic group. In the present paper the notion of an abelian $t$-module replaces that of the algebraic group; and, we will consider both arithmetic and analytic properties of analytic homomorphisms into these objects.

We begin with notation which we will retain throughout this paper:

$\mathbb{F}_{q}$ - a finite field with $q$ elements

$C$ - a smooth projective geometrically irreducible curve over $\mathbb{F}_{q}$

$\infty$ - a fixed closed point of $C$ of degree, $\operatorname{deg}(\infty)$

$k$ - the function field of $C$ over $\mathbb{F}_{q}$

Received by the editors June 29, 1994.

1991 Mathematics Subject Classification. Primary 11G09.

(C)1997 American Mathematical Society 
$A$ - the ring of functions in $k$ which are regular away from $\infty$

$\bar{k}$ - the algebraic closure of $k$

$k_{\infty}$ - the completion of $k$ with respect to the valuation: $-d(a)$ where $d(a)=$ (order of pole of $a$ at $\infty$ ) $\cdot \operatorname{deg}(\infty)$

$\bar{k}_{\infty}$ - the algebraic closure of $k_{\infty}$

Definition 1. Let $\mathbb{G}_{a}$ denote the additive group scheme over a subfield $K$ of $\bar{k}_{\infty}$. Fix an element $t \in A$. A $t$-module of dimension $b$ is a ring homomorphism $\phi$ : $\mathbb{F}_{q}[t] \rightarrow \operatorname{End}_{K}\left(\mathbb{G}_{a}^{b}\right)$ such that:

(1.1) For $a \in \mathbb{F}_{q}, \phi(a) g=a g$ for all $g \in \mathbb{G}_{a}^{b}$.

(1.2) $(d \phi(t)-t I)^{N}$ Lie $\mathbb{G}_{a}^{b}=0$ for some integer $N>0$.

In this context $M_{G}=\operatorname{Hom}_{\mathbb{F}_{q}}\left(\mathbb{G}_{a}^{b}, \mathbb{G}_{a}\right)$ becomes a $\bar{k}_{\infty}[t]$-module via the action

$$
t f=f \circ \phi(t)
$$

If $M_{G}$ is a finitely generated $\bar{k}_{\infty}[t]$-module then $G$ is said to be abelian. Moreover, if there is an integer $r$ such that for all $a \in \mathbb{F}_{q}[t] \operatorname{ker}(\phi(a))$ is a free $\mathbb{F}_{q}[t] /(a)$-module of rank $r$ then $G$ is said to be regular.

For the remainder of this paper we will always assume that the $t$-module is abelian; we drop the adjective "abelian" and refer to an abelian $t$-module simply as a $t$-module. There are well-known examples of one-dimensional $t$-modules which are due to L. Carlitz [Car] and Drinfeld [Dr]. To describe these let $F$ denote the $q$ th-power Frobenius homomorphism and let $K\{F\}$ denote the ring of polynomials in $F$, with coefficients in $K$, with the commutation rule: $F a=a^{q} F$, for $a \in \bar{k}_{\infty}$. Then $\operatorname{Hom}_{K}\left(\mathbb{G}_{a}\right) \cong K\{F\}$. A $t$-module $\phi: \mathbb{F}_{q}[t] \rightarrow K\{F\}$ can then be described by fixing a polynomial $\phi(t) \in K\{F\}$, whose constant term is $t F^{0}$, and then extending the action to all of $\mathbb{F}_{q}[t]$ by linearity. When degree $_{F} \phi(t)=1$, this is the so-called Carlitz module.

An important higher dimensional example has been given by G. Anderson and D. Thakur [An-Th]. For any $n \geq 1$ they have constructed what amounts to an $n$th tensor power of the Carlitz module. This is a simple $t$-module in that it has no proper algebraic subgroups which are closed under the $t$-action. An important arithmetic consequence of their construction (due essentially to $\mathrm{Yu}$ [Yu 1]) is that when $\zeta_{C}(z)$ denotes the Carlitz zeta function, the values $\zeta_{C}(n)$ are transcendental over $k$. (The transcendence of these values is demonstrated by showing that, up to an algebraic multiplicative constant, $\zeta_{C}(n)$ is the last coordinate of a point in the tangent space of the $t$-module, which has algebraic coordinates under the exponential mapping.)

There are other important $t$-modules, the ones with a trival $t$-action, trival meaning that the Frobenius is not involved in the action. If $G=\left(\mathbb{G}_{a}^{n}, \phi\right)$ then

$$
\phi(t)=t I_{n}+M
$$

where $I_{n}$ is the $n \times n$ identity matrix and $M$ is an $n \times n$ nilpotent matrix over $\bar{k}_{\infty} . G$ is not abelian since $\operatorname{Hom}_{\mathbb{F}_{q}}\left(\mathbb{G}_{a}^{n}, \mathbb{G}_{a}\right) \cong M_{n x 1}\left(\mathbb{F}_{q}[F]\right)$ cannot then be finitely generated over $\bar{k}_{\infty}[t]$, as a module, since all powers of $F$ must be included in the generating set. However, this is a regular $t$-module. In the other direction Anderson has shown that all abelian $t$-modules are regular. 


\section{ANALYTIC HOMOMORPHISMS}

1. Throughout this paper we will be interested in analytic homomorphisms, in $m \geq 1$ variables, from $\bar{k}_{\infty}^{m}$ to $G\left(\bar{k}_{\infty}\right)$. To be precise we begin with a definition.

Definition 2. An $\mathbb{F}_{q}$-linear analytic map $\Phi: \bar{k}_{\infty}^{m} \rightarrow G\left(\bar{k}_{\infty}\right)$ is said to be an analytic homomorphism into $G$, defined over $K \subseteq \bar{k}_{\infty}$, if

(2.1) there exists an $m \times m$ matrix $d \phi_{m}(t)$ over $K$ such that

$$
\left(d \phi_{m}(t)-t I\right)^{m}=0
$$

and

$$
\Phi\left(d \phi_{m}(t) \mathbf{z}\right)=\phi(t) \Phi(\mathbf{z})
$$

for all $\mathbf{z} \in \bar{k}_{\infty}^{m}$, and

(2.2) it has a Taylor expansion at the origin of the form

$$
\Phi(\mathbf{z})=\sum_{h=0}^{\infty} \beta_{h} \mathbf{z}^{(h)} \quad \text { for all } \quad \mathbf{z} \in \bar{k}_{\infty}^{m}
$$

with $\beta_{h} \in \operatorname{Mat}_{b \times m}(K)$.

An analytic homomorphism $\Phi: \bar{k}_{\infty}^{m} \rightarrow G\left(\bar{k}_{\infty}\right)$ is also referred to as an $m$ parameter subgroup of $G$.

We will need to understand the relationship between an $m$-parameter subgroup $\Phi(z)$ of $G$ and $G$ 's canonically associated exponential mapping $\exp _{G}: \bar{k}_{\infty}^{b} \rightarrow G\left(\bar{k}_{\infty}\right)$. This relationship is central to the analytic nature of the $m$-parameter subgroup and to the arithmetic nature of its values.

Given two $t$-modules $G=\left(\mathbb{G}_{a}^{b}, \phi\right)$ and $G^{\prime}=\left(\mathbb{G}_{a}^{b^{\prime}}, \phi^{\prime}\right)$ a morphism $f: G \rightarrow G^{\prime}$ of $t$-modules is a morphism of algebraic groups which additionally satisfies:

$$
f \circ \phi(a)=\phi^{\prime}(a) \circ f \quad \forall a \in \mathbb{F}_{q}[t] .
$$

Proposition 3 (Anderson). Let $f: G \rightarrow G^{\prime}$ be a morphism of $t$-modules. Then there exists a unique mapping $\exp _{f}: \operatorname{Lie}(G) \rightarrow G^{\prime}\left(\bar{k}_{\infty}\right)$ such that for all $\mathbf{z} \in \operatorname{Lie}(G)$ and all $a \in \mathbb{F}_{q}[t]$

$$
\exp _{f}(d \phi(a) \mathbf{z})=\phi^{\prime}(a) \circ \exp _{f}(\mathbf{z}) .
$$

Moreover, if we identify $G$ and $G^{\prime}$ with $\bar{k}_{\infty}^{b}$ and $\bar{k}_{\infty}^{b^{\prime}}$ (respectively) by mappings $\kappa$ and $\kappa^{\prime}$, then there exists an entire additive mapping $e: \bar{k}_{\infty}^{b} \rightarrow \bar{k}_{\infty}^{b^{\prime}}$ such that for all $\mathbf{z} \in \operatorname{Lie}(G)$

$$
e\left(\kappa_{*}(\mathbf{z})\right)=\kappa^{\prime} \exp _{f}(\mathbf{z})
$$

Proof. See [An], page 472.

It follows from (1) that there is a canonically associated exponential mapping $\exp _{G}: \operatorname{Lie}(G) \rightarrow G\left(\bar{k}_{\infty}\right)$ which is simply $\exp _{f}$ where $f: G \rightarrow G$ is the identity mapping. Moreover, from (2), the correspondence $G \rightarrow \exp _{G}$ is functorial in that given a morphism $f: G \rightarrow G^{\prime}$ one obtains:

$$
\exp _{G^{\prime}} \circ f_{*}=\exp _{f}=f \circ \exp _{G},
$$

where $f_{*}=\kappa_{*}^{\prime-1} \circ e_{*} \circ \kappa_{*}$.

The central importance of the canonically associated exponential mapping also manifests itself in the following result. 
Proposition 4. If $\Phi: \bar{k}_{\infty}^{m} \rightarrow G\left(\bar{k}_{\infty}\right)$ is an m-parameter subgroup, then there exists a linear mapping $\Phi_{*}: \bar{k}_{\infty}^{m} \rightarrow \bar{k}_{\infty}^{b}$ such that for all $\mathbf{z} \in \bar{k}_{\infty}^{m}$,

$$
\Phi(\mathbf{z})=\exp _{G}\left(\Phi_{*}(\mathbf{z})\right) .
$$

Proof. Let $G^{\prime}$ denote the Zariski closure of $\Phi\left(\bar{k}_{\infty}^{m}\right)$ in $G$. Then $G^{\prime}$ is a $t$-submodule of $G$ which inherits a coordinate system from the coordinate system on $G$. Let $\exp _{G^{\prime}}$ denote the exponential mapping associated with this coordinate system and consider the diagram:

$$
\begin{array}{rlll}
\bar{k}_{\infty}^{m} \stackrel{\Phi}{\longrightarrow} G^{\prime} & \stackrel{i}{\hookrightarrow} & G \\
\searrow \beta_{0} \quad \uparrow \exp _{G^{\prime}} & & \uparrow \exp _{G} \\
& \bar{k}_{\infty}^{n} & \stackrel{i_{*}}{\longrightarrow} & \bar{k}_{\infty}^{b}
\end{array}
$$

Functoriality of the exponential mapping yields: $\exp _{G^{\prime}}(z)=\exp _{G}\left(i_{*} z\right)$. But then the uniqueness of $\exp _{G^{\prime}}$ shows that $\Phi(x)=\exp _{G^{\prime}}\left(\beta_{0} z\right)$. Whence (4).

2. Since both $\Phi(\mathbf{z})$ and $\exp _{G}(\mathbf{z})$ are analytic, i.e. can be represented by everywhere convergent Taylor series, we can use equation (4) to relate the Taylor series coefficients of these two functions. We assume throughout this section that $K$ is a finite extension of $k$.

Suppose that the $t$-module is given by a homomorphism

$$
\phi: \mathbb{F}_{q}[t] \rightarrow \operatorname{End}_{K}\left(\mathbb{G}_{a}^{b}\right) .
$$

Since $\phi$ is a ring homomorphism, it can be realized as a matrix with entries in $K\{F\}$. To fix notation write

$$
\phi(t)=\sum_{j=0}^{r} G_{j} F^{j}
$$

with $G_{j} \in \operatorname{Mat}_{b \times b}(K)$ and $F^{j}$ represents the transpose of the vector $\left(F^{j}, \ldots, F^{j}\right)$. We recall that $\exp _{G}$ may be expressed as

$$
\exp _{G}\left(z_{1}, \ldots, z_{b}\right)=\left(e_{1}(\mathbf{z}), \ldots, e_{b}(\mathbf{z})\right)
$$

where each function $e_{j}: \bar{k}_{\infty}^{b} \rightarrow \bar{k}_{\infty}$ is an $E_{q}$-function with respect to some finite extension $K$ of $k$.

The invariance property of the canonically associated exponential mapping,

$$
\exp _{G}(d \phi(t) \mathbf{z})=\phi(t) \exp _{G}(\mathbf{z})
$$

yields the representation:

$$
\exp _{G}(\mathbf{z})=\left(\begin{array}{c}
z_{1} \\
\vdots \\
z_{b}
\end{array}\right)+\sum_{h=1}^{\infty} A_{h}\left(\begin{array}{c}
z_{1}^{q^{h}} \\
\vdots \\
z_{b}^{q^{h}}
\end{array}\right)
$$

where the $b \times b$ matrices $A_{h}$ satisfy the recursion

$$
\left(t^{q^{h}}-t\right) A_{h}=\sum_{j=1}^{\inf \{h, r\}} G_{j} A_{h-j}^{\left(q^{j}\right)} .
$$

(Here $A_{h-j}^{\left(q^{j}\right)}$ indicates that each entry of $A_{h-j}$ has been raised to the $q^{j}$-th power.) 
Summarizing Lemma 6.1 of [Yu 3] we find that

$$
d\left(A_{h}\right)=\max \left\{d\left(a_{h}\right): a_{h} \text { is an entry in } A_{h}\right\}
$$

satisfies

$$
d\left(A_{h}\right) \leq\left[\frac{1}{C_{1}}\right] h q^{h}
$$

for $h$ sufficiently large, where $C_{1}$ is any constant $C_{1}>\frac{r}{d(t)}$.

This inequality has analytic consequences for the growth of $\exp _{G}$ and arithmetic consequences for its values. On the analytic side we recall that in this setting the maximum modulus principle has a particularly simple form. For an entire function

$$
f\left(z_{1}, \ldots, z_{b}\right)=\sum c_{1} z_{1}^{l_{1}} \ldots z_{b}^{l_{b}}
$$

let $M_{R}(f)=\sup _{\mathbf{1}}\left\{d\left(c_{\mathbf{l}}\right)+\left|l_{1}+\cdots+l_{b}\right| R\right\}$. If for a vector $\mathbf{z}$ we put $d(\mathbf{z})=$ $\max \left\{d\left(z_{1}\right), \ldots, d\left(z_{b}\right)\right\}$ then

$$
\sup _{d(\mathbf{z}) \leq R} d(f(\mathbf{z}))=\sup _{d(\mathbf{z})=R} d(f(\mathbf{z}))=M_{R}(f) .
$$

Specializing this to the component functions $e_{j}(\mathbf{z})$ we apply Lemma 2.4 of [Yu 4] to obtain: For $R$ sufficiently large,

$$
M_{R}\left(e_{j}\right) \leq q^{C_{1} R}
$$

for all $j$. Hence

$$
M_{R}\left(\exp _{G}\right)=\max \left\{M_{R}\left(e_{1}\right), \ldots, M_{R}\left(e_{b}\right)\right\} \leq q^{C_{1} R}
$$

provided $R$ is sufficiently large.

If we express $\Phi_{*}: \bar{k}_{\infty}^{m} \rightarrow \bar{k}_{\infty}^{b}$ by

$$
\Phi_{*}\left(z_{1}, \ldots, z_{m}\right)=P\left(\begin{array}{c}
z_{1} \\
\vdots \\
z_{m}
\end{array}\right),
$$

where $P=\left(\alpha_{i j}\right)$ is a $b \times m$ matrix, then the arithmetic of the matrix entries $\alpha_{i j}$, $1 \leq i \leq b, 1 \leq j \leq m$, will play a role in the arithmetic of the values we are investigating, and, potentially, in the growth of $\Phi$.

To account for this we must establish a size for elements of a finitely generated extension $E$ of a finite extension $F$ of $k$. We recall this, with a slight change of notation, from $[\mathrm{B}-\mathrm{B}-\mathrm{T}]$. Fix a transcendence basis $\left\{\theta_{1}, \ldots, \theta_{s}\right\}$ of $E$ over $F$ and put $E_{\mathrm{tr}}=F\left(\theta_{1}, \ldots, \theta_{s}\right)$. We view $E$ as a vector space over $E_{\mathrm{tr}}$ with a basis $\left\{\eta_{1}(=1), \eta_{2}, \ldots, \eta_{n}\right\}$. We also view $F$ as a vector space over $k$ with an integral basis $\left\{\alpha_{1}(=1), \alpha_{2}, \ldots, \alpha_{f}\right\}$. Let $F_{\phi}$ denote the $\mathbb{F}_{q}[t]$-span of $\alpha_{1}, \ldots, \alpha_{f}$.

Then any element $x \in E$ may be uniquely expressed as:

$$
x=\left(\sum_{\sigma=1}^{n} P_{\sigma}\left(\theta_{1}, \ldots, \theta_{s}\right) \eta_{\sigma}\right) / P_{0}\left(\theta_{1}, \ldots, \theta_{s}\right)
$$

where $P_{0}, P_{1}, \ldots, P_{n}$ have coefficients in $F_{\phi}$. Moreover, we may assume that these polynomials are coprime in the sense that if we write

$$
P_{\sigma}\left(X_{1}, \ldots, X_{s}\right)=\sum_{d_{1}} \cdots \sum_{d_{s}} \sum_{j=1}^{f} a_{i, \mathbf{d}, j} \alpha_{j} X_{1}^{d_{1}} \cdots X_{s}^{d_{s}}
$$

then we may take the coefficients $a_{i, \mathbf{d}, j}$ without any common factors in $\mathbb{F}_{q}[t] \backslash \mathbb{F}_{q}$. 
When $x$ is represented as in (5), we define a degree, $D(x)$, and height, $h(x)$, by

$$
D(x)=\max \left\{\operatorname{deg} P_{0}, \ldots, \operatorname{deg} P_{n}\right\}
$$

(where deg denotes total degree), and

$$
h(x)=\max \left\{d\left(a_{i, \mathbf{d}, j}\right)\right\} .
$$

With these definitions one has:

Proposition 5. There exist constants $C(D)$ and $C(h)$ so that for $x_{1}, \ldots, x_{\ell} \in K$ :

$$
\begin{aligned}
D\left(x_{1} \cdots x_{\ell}\right) & \leq D\left(x_{1}\right)+\cdots+D\left(x_{\ell}\right)+C(D)(\ell-1), \\
h\left(x_{1} \cdots x_{\ell}\right) & \leq h\left(x_{1}\right)+\cdots+h\left(x_{\ell}\right)+C(h)(\ell-1) .
\end{aligned}
$$

Proof. (See $[\mathrm{B}-\mathrm{B}-\mathrm{T}]$.

We now come to the arithmetic of the coefficients of $\Phi(\mathbf{z})$. Assume that $\Phi(\mathbf{z})$ is defined over a field $F$. Recalling the notation

$$
\left(\begin{array}{c}
w_{1}^{q^{h}} \\
\vdots \\
w_{b}^{q^{h}}
\end{array}\right)=\mathbf{w}^{q^{h}}
$$

we then have (with the additional convention that $A_{0}$ is the identity matrix)

$$
\Phi\left(z_{1}, \ldots, z_{m}\right)=\sum_{h=0}^{\infty} A_{h}(P \cdot \mathbf{z})^{q^{h}}
$$

where $P \in \operatorname{Mat}_{b \times m}(F)$. If we write

$$
\Phi\left(z_{1}, \ldots, z_{m}\right)=\left(f_{1}(\mathbf{z}), \ldots, f_{b}(\mathbf{z})\right)
$$

we then have the explicit representations:

$$
\begin{aligned}
f_{j}\left(z_{1}, \ldots, z_{m}\right) & =\sum_{h=0}^{\infty}\left(b_{j 1}^{(h)}, \ldots, b_{j m}^{(h)}\right) \cdot\left(\begin{array}{c}
\sum_{k=1}^{m} \alpha_{1 k} z_{k} \\
\vdots \\
\sum_{k=1}^{m} \alpha_{m k} z_{k}
\end{array}\right)^{q^{h}} \\
& =\sum_{h=0}^{\infty} \sum_{s=1}^{m} b_{j s}^{(h)}\left(\sum_{k=1}^{m} \alpha_{s k} z_{k}\right)^{q^{h}} \\
& =\sum_{k=1}^{m}\left(\sum_{h=0}^{\infty} c_{k}^{(h)} z_{k}^{q^{h}}\right)
\end{aligned}
$$

where $c_{k}^{(h)}=b_{j 1}^{(h)} \alpha_{1 k}^{q^{h}}+\cdots+b_{j m}^{(h)} \alpha_{m k}^{q^{h}}$.

Thus we obtain the following:

Theorem 6. For an analytic homomorphism $\Phi$ as above with coordinate functions $f_{j}(\mathbf{z})$, expressed as in (7), we have the estimates:

(6.1) $D\left(c_{k}^{(h)}\right) \leq q^{h}\left(\max _{1 \leq s \leq m} D\left(\alpha_{s k}\right)+C_{2}\right)$,

(6.2) $h\left(c_{k}^{(h)}\right) \leq \max _{1 \leq s \leq m}\left\{\operatorname{deg}_{\infty} b_{j s}+q^{h} h\left(\alpha_{s k}\right)\right\}+C_{3}$ 
Moreover, if we take $d_{k}^{(h)} \in F_{\phi}\left[\theta_{1}, \ldots, \theta_{s}\right]$ to be a denominator for $c_{k}^{(h)}$ in the sense that $d_{k}^{(h)} c_{k}^{(h)}$ lies in the $F_{\phi}\left[\theta_{1}, \ldots, \theta_{s}\right]$-span of $\eta_{1}, \ldots, \eta_{n}$, we have the estimates

(6.4) $h\left(d_{k}^{(h)}\right) \leq q^{h}\left(C_{5} h+\max _{1 \leq s \leq m} h\left(\alpha_{s k}\right)+C_{6}\right)$,

(6.5) For all $j \leq h, d_{k}^{(h)} c_{k}^{(h)}$ is in the $F_{\phi}\left[\theta_{1}, \ldots, \theta_{s}\right]$-span of $\eta_{1}, \ldots, \eta_{n}$,

(6.6) If $q^{h_{1}}+\cdots+q^{h_{f}} \leq q^{N}$, then $d_{k}^{\left(h_{1}\right)} \cdots d_{k}^{\left(h_{f}\right)} \mid d_{k}^{(N)}$.

We call such a function a (generalized) $E_{q}$-function for the field $K$. We note for future use that 6.2 implies that, asymptotically, $\Phi$ satisfies the same growth estimate as $\exp _{G}$.

We now examine how such a function transforms under the $\mathbb{F}_{q}[t]$-action given by the representation $\phi$. In particular, for later applications we need to understand the Taylor coefficients of the coordinate functions of $\Phi\left(d \phi_{m}(a) \mathbf{z}\right)$, for $a \in \mathbb{F}_{q}[t]$. To be explicit write

$$
\phi(a)=\sum_{j=0}^{r} G_{j}(a) F^{j}
$$

with $G_{j}(a) \in \operatorname{Mat}_{b \times b}(K)$ and $G_{0}(a)=d \phi_{m}(a)$. Thus

$$
\Phi\left(d \phi_{m}(a) \mathbf{z}\right)=\phi(a) \circ \Phi(\mathbf{z})
$$

yields

$$
\Phi\left(d \phi_{m}(a) \mathbf{z}\right)=\left(\sum_{j=0}^{r} G_{j}(a) F^{j}\right) \circ\left(\begin{array}{c}
f_{1}(\mathbf{z}) \\
\vdots \\
f_{b}(\mathbf{z})
\end{array}\right) .
$$

Let $G_{j}(a)=\left(p_{i g}^{(j)}(a)\right)$, then

$$
\Phi\left(d \phi_{m}(a) \mathbf{z}\right)=\sum_{j=0}^{r}\left(\sum_{g=1}^{b} p_{i g}^{(j)}(a) f_{g}^{q^{j}}(\mathbf{z})\right)=\left(\begin{array}{c}
B_{1}(\mathbf{z}) \\
\vdots \\
B_{b}(\mathbf{z})
\end{array}\right) .
$$

From this representation and (8) we obtain explicit expressions for the Taylor coefficients of $\Phi\left(d \phi_{m}(a) \mathbf{z}\right)$. In particular, we have

$$
\begin{aligned}
B_{i}(\mathbf{z}) & =\sum_{j=0}^{r} \sum_{g=1}^{b} p_{i g}^{(j)}(a)\left(\sum_{k=1}^{m}\left(\sum_{h=0}^{\infty} c_{k}^{(h)} z_{k}^{q^{h}}\right)\right)^{q^{j}} \\
& =\sum_{k=1}^{m} \sum_{h=0}^{\infty} \underbrace{\left(\sum_{j=0}^{r} \sum_{g=1}^{b} p_{i g}^{(j)}(a)\left(c_{k}^{h}\right)^{q^{j}}\right)}_{d_{i \ell}^{(k)}} z_{k}^{q^{h}}
\end{aligned}
$$

Thus,

$$
\begin{aligned}
D\left(d_{i h}^{(k)}(a)\right) & \leq q^{r} \max D\left(c_{k}^{(h)}\right)+\max D\left(p_{i g}^{(j)}(a)\right)+C_{7}, \\
h\left(d_{i h}^{(k)}(a)\right) & \leq q^{r}\left(\max h\left(c_{k}^{(h)}\right)+\max h\left(p_{i g}^{(j)}(a)\right)+C_{8}\right) .
\end{aligned}
$$


3. We next suppose that $H$ is an algebraic subgroup of $\mathbb{G}_{a}^{b}$ which is invariant under the action of $\mathbb{F}_{q}[t]$. We say that $H$ is a $t$-submodule of $G=\left(\mathbb{G}_{a}^{b}, \phi\right)$.

Theorem 7. Suppose that $H$ is a $t$-submodule of $G$ such that $G / H$ has no torsion points under the induced t-action. Then $G / H$ is a $t$-module. Moreover, if $\pi: G \rightarrow$ $G / H$ is the canonical projection mapping, then

$$
\exp _{\pi}=\pi \circ \exp _{G}
$$

is an entire $\mathbb{F}_{q}$-linear mapping from LieG to $G / H$.

Proof. Since $G / H$ has no torsion points the induced action is well-defined. That condition (1.1) holds is evident. To verify that (1.2) holds choose $s$ so that (1.2) holds for the $t$-action on $G$ with $N=p^{s}$.

Let $V_{H} \subseteq$ LieG denote the Lie sub-algebra of LieG which is the Lie algebra of $H$. Take a basis of $V_{H}$ and extend it to a basis for all of $L i e G$. If we let $M$ denote the change of basis matrix from the standard basis to this, possibly, different one we obtain

$$
M d \phi(t) M^{-1}=\left(\begin{array}{cc}
A & B \\
0 & C
\end{array}\right),
$$

where $A$ is a $\operatorname{dim} H \times \operatorname{dim} H$ matrix. We then have

$$
\left(M d \phi(t) M^{-1}-t I\right)^{p^{s}} \operatorname{Lie} G=0 .
$$

This implies that $(C-t I)^{p^{s}}$ annihilates $(\operatorname{Lie} G) / V_{H}$, and therefore Lie $(G / H)$.

Then $\pi$ becomes a morphism of $t$-modules and by the functorality of the exponential function as given by (3), $\exp _{\pi}$ is an entire $\mathbb{F}_{q}$-linear mapping.

Remark. By Lemma 1.3 of [Yu 1] the mapping $\pi_{*}$ is surjective. Thus if $\exp _{G}$ is surjective it follows that $\exp _{G / H}$ is surjective. We give an alternate proof of this in the next section.

When $\Phi: \bar{k}_{\infty}^{m} \rightarrow G\left(\bar{k}_{\infty}\right)$ is an $m$-parameter subgroup, we consequently obtain an $m$-parameter subgroup $\widehat{\Phi}: \bar{k}_{\infty}^{m} \rightarrow G / H\left(\bar{k}_{\infty}\right)$. We formalize this as the following theorem.

Theorem 8. Suppose that $H$ is a $t$-submodule of $G$ of dimension b, with $G / H$ being a t-module; and, suppose that $\Phi: \bar{k}_{\infty}^{m} \rightarrow G\left(\bar{k}_{\infty}\right)$ is an m-parameter subgroup of $G$. Let $\pi: G \rightarrow G / H$ denote the canonical projection. Then $\widehat{\Phi}=\pi \circ \Phi$ induces an analytic homomorphism from $\bar{k}_{\infty}^{m}$ to $G / H\left(\bar{k}_{\infty}\right)$. Moreover there is a canonically associated mapping $\pi_{*}: \bar{k}_{\infty}^{b} \rightarrow \bar{k}_{\infty}^{l}$ so that $d \widehat{\Phi}=\pi_{*} \circ d \Phi$.

Proof. Write $\Phi(\mathbf{z})=\exp _{G} \circ \Phi_{*}(\mathbf{z})$. Then Theorem/Definition 3 of [An], Proposition 3 above, tells us that there exists an entire additive mapping $e: G\left(\bar{k}_{\infty}\right) \rightarrow G / H\left(\bar{k}_{\infty}\right)$ so that we obtain the diagram

$$
\begin{array}{r}
\bar{k}_{\infty}^{m} \stackrel{d \Phi}{\longrightarrow} \bar{k}_{\infty}^{b} \stackrel{\exp _{G}}{\longrightarrow} G\left(\bar{k}_{\infty}\right) \\
\searrow \quad \downarrow \pi \\
G / H\left(\bar{k}_{\infty}\right)
\end{array}
$$

where the triangle of maps commutes.

If we let $\exp _{G / H}: \bar{k}_{\infty}^{l} \rightarrow G / H\left(\bar{k}_{\infty}\right)$ denote the exponential mapping of $G / H$ then there exists $\pi_{*}$ so that

$$
\pi \circ \exp _{G}=\exp _{G / H} \circ \pi_{*} .
$$


Hence,

$$
\widehat{\Phi}=\pi \circ \Phi=\pi \circ \exp _{G} \circ \Phi_{*}=\exp _{G / H} \circ \pi_{*} \circ \Phi_{*}
$$

yields the result.

We note that if $G$ and $H$ are defined over a subfield $K$ of $\bar{k}_{\infty}$, then $\pi$ is as well and the Taylor coefficients of $e(z)$, at the origin, will lie in $K$. Hence the field of definition of $\widehat{\Phi}_{*}$ is the same as that of $\Phi_{*}$.

\section{Structure THeOREMS FOR $t$-MOdules}

4. We return to the situation where $K$ is an arbitrary subfield of $\bar{k}_{\infty}$. For a $t$ module $G$ the group of morphisms of $K$-algebraic groups from $G$ to $\mathbb{G}_{a}$ is denoted by $M_{G} ; M_{G}$ is a $t$-motive in the sense of Anderson, [An]. $M_{G}$ is endowed with a $K[t, F]$-module structure such that for $g \in G, k \in K$, and $m \in M_{G}$ :

$$
\begin{aligned}
(k m)(g) & =k(m(g)), \\
(F m)(g) & =m(g)^{q},
\end{aligned}
$$

and

$$
(t m)(g)=m(\phi(t) g) .
$$

One of Anderson's most fundamental results is that the mapping $G \rightarrow M_{G}$ is a functor which establishes an anti-equivalence between the categories of (abelian) $t$-modules and $t$-motives. Consequently, information about $G$ is encoded in $M_{G}$.

For a $t$-module $G$ we put

$$
r(M)=\operatorname{rank}_{K[t]} M_{G} \quad \text { and } \quad p(M)=\operatorname{rank}_{K[F]} M_{G}
$$

The weight of $G$, or of $M_{G}$, is defined to be the ratio $p\left(M_{G}\right) / r\left(M_{G}\right)$. It is denoted by $w t(G)$.

For a large class of $t$-modules this ratio is invariant for submodules and quotient modules. To describe this class we must introduce some additional notation. Let $M=M_{G}$ be a left $K[t, F]$-module as described above. Put

$$
M((1 / t))=M \otimes_{K[t]} K((1 / t)) .
$$

$M((1 / t))$ is a left $K[t, F]$-module under

$$
F\left(m \otimes \sum a_{i} t^{i}\right)=(F m) \otimes \sum a_{i}^{q} t^{i} .
$$

$M$ can be identified as a $K[t, F]$-submodule of $M((1 / t))$.

A subset $L$ of $M((1 / t))$ is said to be a $K[[1 / t]]$-lattice if $L$ is a finitely generated $K[[1 / t]]$-submodule of $M((1 / t))$ which generates $M((1 / t))$ over $K((1 / t))$. Then, $M$ is pure if there exist positive integers $u$ and $v$ such that for some $K[[1 / t]]$-lattice $L$

$$
t^{u} L=F^{v} L \text {. }
$$

Proposition 9 (Anderson). Let $M$ be a pure t-motive and $L$ a $K[[1 / t]]$-lattice with $t^{u} L=F^{v} L$ for some positive integers $u$ and $v$. Suppose further that $M^{\prime}$ is a nonzero $K[t, F]$-submodule of $M$ and $M^{\prime \prime}$ is a nonzero $K[t, F]$-quotient of $M$ without $K[t]$ torsion.

Then

$$
w t(M)=w t\left(M^{\prime}\right)=w t\left(M^{\prime \prime}\right)=u / v .
$$

Proof. This is a restatement of Lemma 1.10.1 and Proposition 1.10.2 of [An]. 
We first show that this category contains many natural examples.

Theorem 10. Let $G_{1}, \ldots, G_{b}$ be Drinfeld modules of rank $r$. Then $G=G_{1} \times \cdots \times$ $G_{b}$ with the diagonal $t$-action is pure with $w t(G)=1 / r$.

Proof. Suppose that for each $i, 1 \leq i \leq b$,

$$
\phi_{i}(t)=t+g_{i 1}(t) F+\cdots+g_{i r}(t) F^{r}
$$

with $g_{i j}(t) \in \mathbb{F}_{q}[t]$. Then the diagonal action is given by

$$
[t] \cdot\left(\begin{array}{c}
x_{1} \\
\vdots \\
x_{b}
\end{array}\right)=\left(\begin{array}{cccc}
\phi_{1}(t) x_{1} & 0 & \cdots & 0 \\
0 & \phi_{2}(t) x_{2} & & \vdots \\
\vdots & & & 0 \\
0 & \cdots & 0 & \phi_{b}(t) x_{b}
\end{array}\right) .
$$

Equip $M_{G}$ with the $K[t, F]$ structure above. Explicitly we have

$$
M_{G} \cong \underbrace{K\{F\} \times \cdots \times K\{F\}}_{b \text {-times }} .
$$

So, for $\mathbf{m}=\left(m_{1}(F), \ldots, m_{b}(F)\right) \in M_{G}$

$$
t \mathbf{m}=\mathbf{m}\left(\begin{array}{ccc}
\phi_{1}(t) & & 0 \\
& \ddots & \\
0 & & \phi_{b}(t)
\end{array}\right)=\mathbf{m} \phi(t) .
$$

Let $\mathbf{m}_{1}, \ldots, \mathbf{m}_{b}$ be the standard basis of $M(G)$ over $K\{F\}$, so that

$$
\left\{\mathbf{m}_{j}, F \mathbf{m}_{j}, \ldots, F^{r} \mathbf{m}_{j}\right\}
$$

will generate $\operatorname{End}_{K}\left(G_{j}, \phi_{j}\right)$ over $K$.

For $j \geq 1$ put

$$
L_{j}=\operatorname{span}_{K[[1 / t]]}\left\{\mathbf{m}_{1}, \ldots, F^{r j} \mathbf{m}_{1}, \mathbf{m}_{2}, \ldots, F^{r j} \mathbf{m}_{2}, \ldots, \mathbf{m}_{b}, \ldots, F^{r j} \mathbf{m}_{b}\right\} .
$$

It is easy to verify that $L_{j}$ is a lattice. Our goal is to show that

$$
t L_{j}=F^{r} L_{j} .
$$

Then our $t$-module is pure; and, by Proposition 9 , has weight $1 / r$.

We begin by noting that

$$
F^{r} L_{j}=\operatorname{span}_{K[[1 / t]]}\left\{F^{r} \mathbf{m}_{1}, \ldots, F^{r(j+1)} \mathbf{m}_{1}, \ldots, F^{r} \mathbf{m}_{b}, \ldots, F^{r(j+1)} \mathbf{m}_{b}\right\},
$$

and hence from the representations $\phi_{j}(t)=d \phi_{j}(t) F^{0}+g_{j 1} F+\cdots+g_{j r} F^{r}$ we obtain the inclusion $L_{j} \subset F^{r} L_{j}$.

Therefore, as in the proof of Proposition 4.1.1 of [An] we have

$$
t L_{j}=L_{j+1}=F^{r} L_{j}+L_{j}=F^{r} L_{j} .
$$

Remark. We note that if $G=G_{1} \times \cdots \times G_{b}$ is a product of Drinfeld modules of ranks $r_{1}, \ldots, r_{b}$, which is endowed with the diagonal action, then $G$ is a $t$-module of weight $b /\left(r_{1}+\cdots+r_{b}\right)$. This follows from the easy estimates:

$$
\operatorname{rank}_{K[t]} M_{G}=r_{1}+\cdots+r_{b}, \quad \text { and, } \operatorname{rank}_{K[F]} M_{G}=b .
$$

For applications involving the algebraic independence of several (or even many) values associated with analytic subgroups of $t$-modules the image of the analytic 
subgroup must be understood. Thanks to Proposition 4 it is basic that the image of the canonically associated exponential mapping be understood.

Theorem 4 of [An] gives equivalent formulations for the surjectivity of the exponential mapping. One of these is in terms of the $\mathbb{F}_{q}[t]$-rank of ker $\exp _{G}$. The other involves the concept of a rigid-analytically trivial $t$-module. This requires a definition.

Let

$$
\begin{aligned}
\bar{k}_{\infty}\{t\}= & \left\{\sum_{i=0}^{\infty} a_{i} t^{i}: a_{i} \in \bar{k}_{\infty}, \lim _{i \rightarrow \infty}\left|a_{i}\right|=0, \quad\right. \text { and } \\
& \left.\exists d \text { s.t. } \operatorname{deg}\left(a_{i}\right) \leq d \text { for all } i=1,2, \ldots\right\} .
\end{aligned}
$$

Put

$$
M_{G}\{t\}=M_{G} \otimes_{\bar{k}_{\infty}[t]} \bar{k}_{\infty}\{t\},
$$

which is a left $\bar{k}_{\infty}[t, F]$ module, with $M_{G}\{t\}^{F}$ denoting the elements which are fixed by $F$ under the action

$$
F\left(m \otimes \sum a_{i} t^{i}\right)=(F \circ m) \otimes \sum a_{i}^{q} t^{i}
$$

Definition 11. $G$ is rigid-analytically trivial if the free $\bar{k}_{\infty}\{t\}$-module $M_{G}\{t\}$ has a basis consisting of fixed points of $F$.

An alternate formulation of this definition is given as follows: consider the mapping

$$
M_{G}\{t\}^{F} \otimes_{\mathbb{F}_{q}[t]} \bar{k}_{\infty}\{t\} \stackrel{\rho}{\rightarrow} M_{G} \otimes_{\bar{k}_{\infty}[t]} \bar{k}_{\infty}\{t\}
$$

given by

$$
\left(m \otimes_{\bar{k}_{\infty}[t]} \sum b_{i} t^{i}\right) \otimes_{\mathbb{F}_{q}[t]} \sum a_{i} t^{i} \mapsto\left(m \otimes_{\bar{k}_{\infty}[t]}\left(\sum b_{i} t^{i}\right)\left(\sum a_{i} t^{i}\right)\right) .
$$

Then $G$ is rigid-analytically trivial precisely when $\rho$ is an isomorphism. With this definition we can now state Anderson's criteria for the surjectivity of the canonically associated exponential mapping.

Proposition 12 ([An], Theorem 4). The following properties of a $t$-module are equivalent:

(12.1) $\operatorname{rank}_{\mathbb{F}_{q}[t]} \operatorname{ker} \exp _{G}=r(G)$,

(12.2) $\exp _{G}$ is surjective,

(12.3) $G$ is rigid-analytically trivial.

As with the notion of purity in the previous section we have the following theorem.

Theorem 13. Let $G$ be a rigid-analytically trivial $t$-module. Suppose that $G^{\prime}$ is a $t$-submodule of $G$ such that $G / G^{\prime}$ is a $t$-submodule. Then both $G^{\prime}$ and $G / G^{\prime}$ are rigid-analytically trivial.

Proof. $\mathrm{Yu}$ in [ $\mathrm{Yu}$ 3], Proposition 5.3 has already shown that $G^{\prime}$ is rigid-analytically trivial. Hence it suffices to show that $G / G^{\prime}$ is as well. Consider the short exact sequence

$$
0 \rightarrow G^{\prime} \hookrightarrow G \stackrel{\pi}{\rightarrow} G / G_{1} \rightarrow 0
$$


Recalling that $M_{G}=\operatorname{Hom}\left(G\left(\bar{k}_{\infty}\right), \mathbb{G}_{a}\left(\bar{k}_{\infty}\right)\right)$ and that $\operatorname{Hom}(\cdot, X)$ is a left-exact contravariant functor we obtain

$$
0 \rightarrow M_{G / G^{\prime}} \rightarrow M_{G} \stackrel{p}{\rightarrow} M_{G^{\prime}}
$$

Moreover, Lemma 5.2 of [Yu 3] implies that $p$ is surjective. Then tensoring with $\bar{k}_{\infty}\{t\}$ over $\bar{k}_{\infty}[t]$, and noting that $\bar{k}_{\infty}\{t\}$ is a flat $\bar{k}_{\infty}[t]-$ module, yields the exact sequence

$$
0 \rightarrow M_{G / G^{\prime}}\{t\} \rightarrow M_{G}\{t\} \rightarrow M_{G^{\prime}}\{t\} \rightarrow 0
$$

Let

$$
M_{1}=\left\{\psi \in M_{G}\{t\}^{F}:\left.\psi\right|_{G^{\prime}}=0\right\}
$$

Recalling that $G$ and $G^{\prime}$ are rigid-analytically trivial and that the restriction mapping

$$
M_{G}\{t\}^{F} \otimes_{\mathbb{F}_{q}[t]} \bar{k}_{\infty}\{t\} \stackrel{\text { res }}{\longrightarrow} M_{G^{\prime}}\{t\}^{F} \otimes_{\mathbb{F}_{q}[t]} \bar{k}_{\infty}\{t\}
$$

is surjective we obtain the commutative diagram:

$$
\begin{aligned}
& 0 \longrightarrow M_{G / G^{\prime}}\{t\} \longrightarrow \quad M_{G}\{t\} \longrightarrow \quad M_{G^{\prime}}\{t\} \longrightarrow 0 \\
& \uparrow i \quad \uparrow \cong \quad \uparrow \cong \\
& 0 \longrightarrow M_{1} \otimes_{\mathbb{F}_{q}[t]} \bar{k}_{\infty}\{t\} \hookrightarrow M_{G}\{t\}^{F} \otimes_{\mathbb{F}_{q}[t]} \bar{k}_{\infty}\{t\} \stackrel{\text { res }}{\longrightarrow} M_{G^{\prime}}\{t\}^{F} \otimes_{\mathbb{F}_{q}[t]} \bar{k}_{\infty}\{t\} \longrightarrow 0
\end{aligned}
$$

It follows that $i$ is an isomorphism.

Hence $M_{G / G^{\prime}}\{t\}$ has a basis consisting of fixed points for the action of $F$; and $G / G^{\prime}$ is, therefore, rigid-analytically trivial.

Explicit examples of pure, rigid-analytically trivial $t$-modules abound. It is one of the main results of Anderson [An] that a pure, rigid-analytically trivial $t$-module corresponds to a scattering matrix. (See $\S 3$ of $[\mathrm{An}]$ for definitions and details.) On a more elementary level any one-dimensional $t$-module is rigid-analytically trivial (as pointed out by Anderson in Proposition 4.1.1, [An]). This is a manifestation of the following fact from non-Archimedean analysis:

Lemma 14. Suppose that $f(z)=\sum_{i=0}^{\infty} a_{i} z^{i}$ is a convergent power series with coeffcients in $k_{\infty}$. Then $f\left(\bar{k}_{\infty}\right) \subseteq \bar{k}_{\infty}$ and $f: \bar{k}_{\infty} \rightarrow \bar{k}_{\infty}$ is surjective.

Proof. For any $a \in \bar{k}_{\infty}, f(a)$ lies in the complete field $k_{\infty}(a) \subseteq \bar{k}_{\infty}$. Moreover, the function $g(z)=f(z)-a$ has zeros and they must lie in $\bar{k}_{\infty}$ since $g(z)$ has its coefficients in $k_{\infty}(a)$.

Theorem 15. A product of rigid-analytically trivial t-modules with the diagonal $t$-action is rigid-analytically trivial.

Proof. Suppose $G=G_{1} \times \cdots \times G_{b}$ where each $G_{i}$ is a rigid-analytically trivial $t$-module. Then $\exp _{G}\left(z_{1}, \ldots, z_{b}\right)=\left(\exp _{G_{1}}\left(z_{1}\right), \ldots, \exp _{G_{b}}\left(z_{b}\right)\right)$ since the $t$-action is diagonal. As each of $\exp _{G_{i}}: \bar{k}_{\infty} \rightarrow G_{i}\left(\bar{k}_{\infty}\right)$ is surjective, $\exp _{G}$ is.

(For a remarkably simple example of a $t$-module which does not have a surjective exponential mapping see $\S 2.2$ of $[\mathrm{An}]$.) 


\section{REFERENCES}

[An] G. Anderson, t-motives, Duke Math. J. 53 (1986), 457-502. MR 87j:30039

[An-Th] G. Anderson and D. Thakur, Tensor powers of the Carlitz module and zeta values, Ann. of Math. 132 (1990), 159-191. MR 91h:11046

[B-B-T] W. D. Brownawell, P. G. Becker, and R. Tubbs, Gelfond's Theorem for Drinfeld modules, Michigan Math. J. 41 (1994), 219-233. MR 95h:11067

[Dr] V. G. Drinfeld, Elliptic modules, Math. Sbornik 94 (1974), translation in 23 (1974), No. 4, 561-592. MR 52:5580

[Car] L. Carlitz, On certain functions connected with polynomials in a Galois field, Duke Math. J. 1 (1935), 137-168.

[Yu 1] J. Yu, Transcendence and special zeta values in characteristic p, Ann. of Math. 134 (1991), 1-23. MR 92g:11075

[Yu 2] J. Yu, Analytic homomorphisms into Drinfeld modules, manuscript, 1991, 16 pp.

[Yu 3] J. Yu, Transcendence and Drinfeld modules: several variables, Duke Math. J. 58 (1989), 559-575. MR 90j: 11056

[Yu 4] J. Yu, Transcendence theory over function fields, Duke Math J. 52 (1985), 517-527. MR 87c:11060a

Department of Mathematics, University of Colorado, Boulder, Colorado 80309

E-mail address: tubbs@euclid.colorado.edu 